\title{
Beitrag zur Kenntnifs der Lucaniden-Fauna von Sumatra.
}

Von

Senator G. Albers zu Hannover.

In einer mir unlängst zur Bestimmung zugegangenen Sammlung von Lucaniden, welche mit zahlreichen Käfern aus anderen Familien bei Sipereck auf Sumatra von einem Missionär Hanstein gesammelt worden, befanden sich auch einige neue Arten, die zwar nicht sehr ansehnlich, jedoch durch andere Eigenschaften nicht uninteressant sind. Von bekannten Arten enthielt die Sammlung aufserdem Stücke des Hexarthrius Deyrollei Parry, des Dorcus Parryi Thomson, des Aegus ogivus Deyrolle und des Aegus malaccus Thomson.

\section{Prosopocoelus forficatus.}

Unicolor, dilute castaneus, subnitidus, supra subtilissime (elytrorum dorso obsoletius, hic inde nitidior) granulosus, capite post oculos tuberculo forti, dentiformi, acuto instructo, mandibulis forficiformibus, basi erectis, deinde deplanatis, intus basi angulatis, deinde excavatis, postea rectis, usque ad apicem acutam denticulatis, prosterno inter coxas postea valde descendente, acuto, tibiis rectis, anticis extus sexies dentatis, dentibus tribus apicalibus connatis, protractis, tibiis posterioribus extus unispinosis. Long. $\sigma^{\star} 13$ mill. (femina ignota).

Dieser kleine, wegen der an der Basis nach oben gebogenen scheerenförmigen Mandibeln und der gedornten Wangen in die Gruppe des Prosopocoelus Oweni Hope und Wimberlyi Parry gehörende kleine Lucanide ist in der Körperform einem kleinen Prosopocoelus sericeus Westw. nicht unähnlich, jedoch durch die haarlose Oberseite und die helle kastanienbraune Färbung sofort zu unterscheiden. Die Vorderwinkel des vorn tief ausgebuchteten Kopfes sind nicht vorspringend, schräg abgestutzt, mit abgerundeten Ecken, die dornförmigen Wangenhöcker nach vorn weniger scharf abgesetzt, als bei Wimberlyi, mehr wie bei Oweni gestaltet. Die schmale herabhängende Oberlippe ist ohne besondere Auszeichnung und an der Spitze einfach abgerundet. Der den Kopf an Breite 
übertreffende Vorderrücken ist vorn an den Seiten rasch, fast bogenförmig erweitert, so dals man die Vorderwinkel fast abgestutzt nennen könnte, dann parallelseitig mit fast geraden und ein wenig ausgeschweiften Seiten. Die Hinterwinkel sind schräg abgestutzt mit abgerundeten Hinter- und in einem kleinen spitzen Zahn ausgezogenen Vorderecken. Die eiförmig gestalteten Fld. sind breiter als der Vorderrücken. Die dem Kopfe etwa an Länge gleichkommenden Mandibeln sind aufsen gerundet, an der Innenseite zunächst der Basis stark ausgebuchtet, etwa wie bei Oweni und stärker als bei Wimberlyi. Der nach unten gerichtete Basalzahn ist nur durch eine starke Winkelung angedeutet. An der Basis der geraden sägezähnigen Endschneide steht noch ein kleines, mehr zurückgezogenes und nach oben gerichtetes zahnförmiges Höckerchen. Der unterhalb der Schneide stehende, bei Oweni und Wimberlyi kräftig entwickelte, nach vorn und unten gerichtete $Z$ ahn, der übrigens bei der Betrachtung von oben durch die Schneide dem Auge entzogen wird, ist hier auch vorhanden, jedoch nur durch ein Höckerchen angedeutet. Sollte der Käfer auch in gröfseren Stücken vorkommen, so ist wohl anzunehmen, dafs alle die zahnartigen Vorsprünge stärker entwickelt sein werden. An der Vorderbrust fällt die kräftige Entwickelung des Prosternalkiels auf, der nach hinten in eine lange herabhängende Spitze ausgezogen ist. Die Vorderschienen sind dadurch ausgezeichnet, dafs der gewöhnliche, lang ausgezogene Gabelzahn am Ende auf seinem Halse noch den zweiten, der fünf scharfen Seitenzähne trägt, während die übrigen recht deutlichen $Z$ Zähnchen sich in fast gleichen $Z$ wischenräumen über die sonstige Aufsenkante der Schienen vertheilen. Der Dorn auf der Aufsenkante der vier hinteren Schienen ist recht deutlich, wenn auch an den hintersten Schienen wie gewöhnlich etwas schwächer entwickelt.

Die Sculptur der Oberseite besteht aus einer äufserst feinen Granulirung, die nur auf den Scheitel und der Mitte der Fld. allmählig erlischt, weshalb diese Stellen glänzender erscheinen. In diese Granulirung mischen sich nur auf dem Kopfe neben und hinter den Augen deutliche Punkte.

Ein einzelnes Männchen jetzt in meiner Sammlung.

\section{Eurytrachelus Alcides Vollenh.}

Hr. Ritsema hat mich schon vor Jahr und Tag darauf aufmerksam gemacht, dafs der von mir (siehe diese Zeitschrift, Jahrg. 1886, p. 83) für Eurytrachelus eurycephalus gehaltene Käfer nicht diese 
Art sei, sondern nur eine massive andere Form des Alcides sein möchte. Diese Vermuthung ward darauf gestützt, dafs das Leydener Museum beide in derselben Originalsendungen erhalten habe und darunter einmal auch ein kleineres Stück, dessen rechte Mandibel ganz die Mandibelform des echten Alcides habe, während die linke Mandibel statt der allmähligen Erweiterung in der Mandibelmitte hier einen kräftig entwickelten Zahn zeige. Ich habe seitdem ein ähnliches Stück gesehen, welches im Uebrigen keinen Unterschied von dem gewöhnlichen Alcides zeigte. Auch habe ich kürzlich ein Stück des eurycephalus von Szibodas auf Java kennen gelernt, welches der von van Vollenhoven (Tydschr. voor Entomologie 1865, Pl. 10, fig. 4) gegebenen Abbildung völlig entspricht, d. h. Mandibeln hat, welche fast ebensolang als Kopf und Halsschild zusammen sind und bei welchen der Mittelzahn nicht stärker als der Basalzahn entwickelt ist. Dals dieser Eurytrachelus von dem Alcides specifisch verschieden ist, kann wohl nicht $\mathrm{zweifelhaft}$ sein, da bei ihm die Oberlippe viel breiter und ihr Vorderrand viel weniger dreieckig, vielmehr bogenförmig ausgebuchtet ist. Auch ist der Kopf in der Stirngegend stärker aufgequollen, so dafs ihm die mittlere Ausbuchtung, wie sie bei Alcides an dem abfallenden Stirnrande vorhanden ist, fehlt.

Wenn Hr. Ritsema damals die Vermuthung ausgesprochen hat, dafs solches eine dem eigentlichen Alcides entsprechende Form des echten eurycephalus von Java sein möchte, so kann ich mich dem nur anschliefsen, indem ich zugleich anerkenne, dafs mein vermeintlicher eurycephalus von Sumatra nur eine zweite Form des Alcides sein dürfte. Die hier fragliche Sammlung enthielt ebenfalls beide Formen. Auch enthielt dieselbe eine Anzahl Weibchen.

Diese, deren Abbildung bei van Vollenhoven völlig verfehlt ist, sind den Weibchen von Eurytrachelus Titanus zum Verwechseln ähnlich, nur ist bei ihnen die Sculptur namentlich auf den Fld. noch schwächer und die Behaarung auf denselben noch weniger wahrnehmbar, so dals sie nur an den Flügeldeckenenden bei heller, schräg einfallenden Beleuchtung einigermalsen deutlich erkannt werden kann. Da beide Käfer aber verschiedenen Gruppen angehören, von denen die eine, nämlich die des Titanus, durch die Haarpinsel auf dem fünften und dem siebenten Fühlergliede charakterisirt ist, eine Auszeichnung, von welcher auch die Weibchen, wenn auch nicht in augenfälliger Weise theilnehmen, so bietet die Vergleichung der Behaarung des siebenten Fühlergliedes, wenigstens 
bei frischen, gut erhaltenen Exemplaren ein absolut sicheres Unterscheidungsmerkmal dar. Bei den Weibchen des Alcides sind nämlich die an der Spitze des siebenten Fühlergliedes stehenden zarten Borstenhärchen insofern kürzer als das achte Fühlerglied, als sie dieses (das erste Glied des Fühlerkammes) nicht überragen, während sie beim Weibchen des Titanus erheblich länger sind.

\section{Eurytrachelus Hansteini n. sp.}

Niger, parallelus, subopacus, capite thorace vix angustiore, hoc elytris paulo latiore, mundibulis, capite prothoraceque subtilissime scabrosis, elytris aperte, disco remotius, lateribus densissime punctatis, mandibulis basi extus curvatis, deinde porrectis, paulo flexuosoemarginatis, apice recurvis, intus versus apicem dente didymo fortiore porrecto, ante apicem dente minuto instructis, apicibus acutis; labri carina modice porrecta, in majoribus contracta, emarginata, angulis acutis; frontis carina parum elevata, fere recta, . medio perparum protracta; prothoracis lateribus antice flexuosoemarginatis, versura postica dentiformi, angulis posticis oblique truncatis, versura postica rotundata, antica subdentata, acuta; tibiis posterioribus (posticis obsoletius) unispinosis. - Long. o o $^{3} 30-47$ mill., 우 27-29 mill.

Dem Eurytr. Reichei Hope nahe verwandt, indem sich bei ihm dieselbe Mandibelform und die gleiche Oberlippenbildung wiederholt, vielleicht aber noch mehr dem Eurytr. Castelnaudi Deyrolle. Von letzterem ist jedoch die grölste Form mit der gleichen Mandibelgestaltung wie bei der var. maxima des Reichei noch nicht bekannt geworden. Was ich selbst davon gesehen, entsprach nur dem von Hrn. Deyrolle beschriebenen und abgebildeten Stücke. Uebrigens ist der Käfer ganz schwarz, viel kleiner als Eurytr. Reichei und auf den. Fld. viel deutlicher, ja recht augenfällig punktirt und hierdurch leicht zu unterscheiden. Dabei zeigt er dieselbe Eigenthümlichkeit in der Bildung der Oberlippe wie Reichei, welche übrigens auch sonst wohl, wie z. B. bei Eurytrachelus Tityus Hope, wenn auch in geringerem Umfange, vorkommt, dafs nämlich der Querkiel der Oberlippe, welcher bei den kleinsten Stücken die ganze Breite der Oberlippe einnimmt, sich, je gröfser die Stücke werden, von den Seiten ab zusammenzieht und dadurch im Verhältnifs zur Gröfse der Stücke immer schmäler wird. Der Querkiel auf dem Vorderkopfe über den Mandibeln ist weniger gerade als bei Reichei, vielmehr in der Mitte etwas bogig vorgezogen. 
Dahinter befindet sich ein schwacher dreieckiger Eindruck, der durch seine tiefschwarze, matte Färbung besonders hervortritt und auch bei den kleinsten Stücken noch sichtbar bleibt. Die kleinsten Stücke haben einfach sichelförmig gekrümmte Mandibeln nit einem einzelnen, dreieckigen, etwas aufgerichteten Zahn mit fast zurückgekrümmter Spitze noch etwas diesseits der Mitte. Die geschweifte Ausbuchtung an den Seiten des Vorderrückens, welche bei dem gröfsten mir vorliegenden Stücke weit stärker als bei Reichei entwickelt ist, und bis über die Mitte hinaus reicht, verschwindet hier völlig. Die feine Granulirung auf Kopf und Vorderrücken ist jedoch nicht völlig geschwunden, nur noch zarter geworden. Dabei tritt eine Punktirung auf dem Kopfe und Vorderrücken namentlich an den Seiten deutlicher hervor. Auf den Fld. zeigt sich eine Rippenbildung, die sich erst hinter der Mitte verliert, so dafs man zwischen Naht und Schulterbuckel sieben deutliche Rippen zählt. Diese Rippen sind fast überall gleichbreit. Die Mittelstücke zeigen alle Zwischenstufen zwischen den Extremen der gestreiften und glatten Fld. Bei ihnen verlieren, was namentlich die Mandibeln betrifft, diese den kleinen Zahn vor der Spitze, der Doppelzahn verbreitert sich und rückt namentlich mit seiner hinteren Spitze nach der Basis zu bis zur Mitte herab. Dann verschwindet seine vordere Spitze und es zeigt sich nun nur noch ein breiter, einfacher, dreieckiger Zahn, dessen längere Vorderseite bei den grölseren Stücken an der Basis als Rest der vorderen Zahnspitze nur noch eine kleine Winkelung zeigt.

Das glänzende Weibchen hat analog den kleinsten Männchen

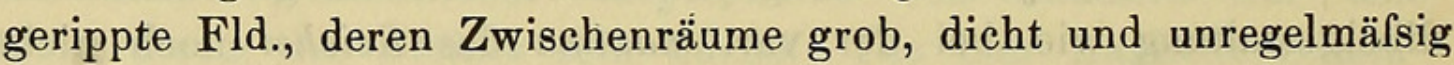
punktirt sind. Die Punkte fliefsen runzlich zusammen und gehen ab und an auf die Rippen über. Der Kopf ist grob runzlich punktirt. Die beiden Kopfhöcker sind gut entwickelt. Die grobe runzliche Punktirung an den Seiten des glänzenden Vorderrückens wird nach der Mitte zu feiner, ohne jedoch ganz zu verschwinden. Nur eine schmale unregelmälsige, den Vorder- und Hinterrand nicht erreichende Mittellinie bleibt punktfrei, während die Punkte neben ihr wieder etwas stärker sind.

Stücke aller Formen in meiner Sammlung.

Gnaphaloryx taurus Fbr.

Dafs der Käfer von Sumatra von dem javanischen Käfer, der gewöhnlich für den taurus genommen wird, specifisch verschieden ist, kann meines Erachtens nicht $\mathbf{z w e i f e l h a f t ~ s e i n , ~ d a , ~ a b g e s e h e n ~}$ 
von dem Unterschiede in den Mandibeln, welche bei den Sumatrakäfern stärker gekrümmt sind und den zweiten Zahn bei grölseren Stücken dicht vor dem Gabelzahne am Ende haben, die Oberlippe fast doppelt so breit als bei dem javanischen Käfer ist. Wenngleich nun aus der Fabricius'schen Beschreibung nicht mit Sicherheit entnommen werden kann, welche Art Fabricius vor sich gehabt hat, indem namentlich die eigentlich auf keinen der beiden Käfer passende Beschreibung der Mandibeln: mandibulae exsertae, intus dentibus duobus validis approximatis, obtusis, darüber in Zweifel lälst, welcher der dritten Zähne, ob der an der Spitze oder der an der Basis übersehen oder abnormer Weise nicht vorhanden gewesen ist, so mufs doch wohl angenommen werden, dafs Fabricius den Sumatrakäfer vor sich gehabt haben werde, da er Sumatra als Vaterland seines Käfers angiebt. Ich möchte daher vorschlagen, für den Sumatrakäfer den Namen taurus beizubehalten und für den javanischen Käfer den Burmeister'schen Namen opacus eintreten zu lassen, da Burmeister (Handbuch V, p. 397) ohne allen

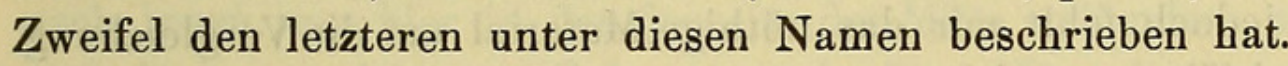

Uebrigens ist die Stellung des zweiten Mandibelzahnes bei dem Sumatrakäfer nicht immer dieselbe; schon bei mittleren Stücken von etwa 30 mill. Länge tritt er bis zur Mitte, ja bisweilen bis diesseits der Mitte zurück, ohne jedoch selbst bei den kleinsten Stücken (mir liegt ein solches von nur 21 mill. Länge vor) vor den Basalzahn so nahe zu treten, als bei dem opacus. Gleicht nun auch der taurus in der Mandibelform, der Zahnstellungen der Mandibeln und der Breite der Oberlippe dem brevis Kirsch. von Neu-Guinea und meinem rugosus von Celebes ganz aufserordentlich, so ist er doch von beiden, abgesehen von sonstigen Unterschieden, wie z. B. die viel schwächere Punktirung auf dem Kopfe, dadurch sicher zu unterscheiden, dafs bei ihm der Kopf trotz seiner Breite und der starken Entwickelung der Wangenhöcker selbst bei den grölsten Stücken entschieden schmäler ist, als der Vorderrücken, während er bei grölseren Stücken der genannten Arten deutlich breiter ist, als der Vorderrücken.

Vom Weibchen hat mir nur ein Stück vorgelegen. Dasselbe gleicht dem des rugosus sehr, ist wie dieses oben anscheinend nackt, doch können wenigstens die zarten feinen Borsten an den Enden der Fld. bei schräg einfallendem Lichte wohl schon mit blofsem Auge wahrgenommen werden; auch fehlen ihm die Spuren der beiden Rippen auf den Fld. zwischen Schulterbuckel und Naht, die bei dem rugosus-Weibchen deutlich hervortreten, völlig. 
Bei dieser Gelegenheit mag ich es übrigens nicht übergehen, darauf aufmerksam zu machen, dafs die Oberlippe bei Gnaphaloryx taurus und seinen obenerwähnten Verwandten ganz eigenthümlich gestaltet ist.

Bei oberflächlicher Beobachtung erscheint sie ähnlich wie bei den Eurytrachelus-Arten gestaltet zu sein, also einen vorspringenden Querkiel zu besitzen, dessen Seitenecken spitz vorspringen, während der Raum dazwischen ausgebuchtet ist. Das ist nun aber durchaus nicht der Fall. Die zahnartigen Spitzen, die wir an den Seiten der Oberlippe wahrnehmen, sind ihre eigenen Seitenecken, was wir bei der Betrachtung dazwischen sehen, ist die Oberfläche der nach unten in eine stumpfe und eingezogene Spitze verlängerten Oberlippe selbst nebst den Anfängen des verdickten Randes, mit welchem die Oberlippe, von den Seitenzähnen ausgehend, eingefafst ist.

Diese Bildungseigenthümlichkeit könnte es vielleicht rechtfertigen, die fraglichen Arten von Gnaphaloryx generisch abzusondern, jedoch fehlt mir das nöthige Material um die Vergleichung ganz durchführen zu können.

\section{Aegus fornicatus n. sp.}

Niger, subnitidus, capitis margine antico emarginato, in medio tuberculis duobus approximatis instructo, post oculos tuberculo rotundato-truncato, mandibulis falcatis, basi extus angulatis alque carinatis, intus basi dente lato, obtuso, apice inciso, post medium dente valido, suberecto et porrecto instructis, prothorace angulis anticis oblique truncatis, emarginatis, posticis oblique undatim emarginatis, versura postica rectangulari, antica obtusa, elytris septies striatis interstitiis obsolete sparsim punctatis, tibiis intermediis extus 3-4 spinosis, posticis unispinosis. - Long. ठ 44 mill. (femina ignota).

Mandibeln, Kopf und Vorderrücken sind äufserst fein granulirt, erstere auf der Oberfläche nur an der Basis deutlich fein punktirt, die Punktirung auf der Fläche des Kopfes und des Vorderrückens so zart, dafs sie mit blofsem Auge nicht wahrgenommen werden kann. Auf ersterem geht sie erst neben und hinter den Augen in eine grobgrubige Punktirung über. Dasselbe findet auf dem Vorderrücken statt, indem die grobe Punktirung am Vorder- und Hinterrande erst in einiger Entfernung von der Mitte beginnt, sich in äufserst geringer Breite an der Randfurche hinzieht und sich nur an den Vorder- und Hinterwinkeln weiter ausbreitet. Auf den 
Zwischenräumen der siebenmal gestreiften Fld. ist nirgend eine reihenweise Anordnung der äufserst feinen Punkte wahrnehmbar. Der Raum zwischen dem siebenten Rückenstreifen und dem Aufsenrande ist deutlich, auch dem blofsen Auge wahrnehmbar punktirt, bleibt jedoch glänzend. Das stark, fast zweilappig ausgebuchtete Kinn ist besonders auf dem Lappen grob grubig punktirt, die übrige Unterseite des Kopfes nur deutlich auf den beiderseitigen Räumen zwischen der Kehle und den Fühlergruben. Dagegen ist das ganze Prosternum zwischen, vor und hinter den Vorderhüften bis auf die eingedrückten Räume neben den Vorderecken grob runzelig punktirt. Die Punktirung auf der Mittelbrust bleibt namentlich auf der Mitte, schwächer. Auf den Hinterleibsringen ist die Punktirung noch feiner, aber mehr gedrängt.

Die Mandibeln haben aufsen an der Basis eine scharfe, fast spitzwinkelige Ecke, sind von da an neben dem Aufsenrande mit allmählig abnehmender Tiefe eingedrückt. Der Aufsenrand erscheint dadurch kielförmig erhaben. Die Erhebung wendet sich alsbald nach innen, um hier in den jenseits der Mitte stehenden, nach vorn und innen gerichteten Zahn auszulaufen. Der breite, oblonge Basalzahn ist in der Mitte der abgestutzten Spitze leicht eingeschnitten, so dafs zwei Knötchen entstehen, welche jedoch mit ihren äufseren Ecken am weitesten vorspringen. Die beiden Knötchen an dem tief ausgebuchteten Vorderrande des Kopfes stehen ziemlich nahe bei einander und sind nur durch eine Ausbuchtung getrennt, deren äufserste Breite etwa der Basisbreite der Höcker entspricht. Bei geschlossenen Mandibeln trifft die Spitze der Höcker mit den Endwinkeln des Basalzahnes an den Mandibeln zusammen. Die Augenkiele sind gerade, nicht ausgebuchtet. Die breiten, flachen, nicht sehr weit vorspringenden Wangenhöcker sind stumpf abgerundet. Die Abrundung ist jedoch keine ganz regelmäfsige, vielmehr vorn etwas mehr eckig als hinten. Die Vorderwinkel des den Kopf an Breite etwas übertreffenden Vorderrückens sind schräg abgestutzt und ausgebuchtet, die Seiten gerade, nach hinten nur äufserst schwach convergirend, die Hinterwinkel, wenn auch nicht tief, doch deutlich wogenförmig ${ }^{1}$ ) ausgebuchtet; die

1) So, d. h. deutsch: wogenförmig, lateinisch: undata, adverb. undatim, glaube ich zweckmäfsig die eigenthümliche Ausbuchtung, wie sie sich so oft bei den Lucaniden an den Hinter- und Vorderwinkeln des Vorderrückens befindet, bezeichnen zu dürfen. Burmeister nennt sie S-förmig, was nicht wohl angeht, weil diese 
hintere dadurch gebildete Ecke ist nicht völlig rechtwinklig, die Vorderecke stumpfwinkelig.

Die Fld. sind schmäler als der Vorderrücken. Die 3-4 Dornen an den Mittelschienen sind recht deutlich, dagegen ist an den Hinterschienen nur ein schwacher Dorn wahrnehmbar.

Besonders ausgezeichnet ist übrigens der Käfer dadurch, dals das erste Glied der Fühlerkeule mit seiner äufseren Vorderecke nach aufsen spitz vorspringt und das folgende Glied nicht nur an seiner Basis, sondern auch an seiner Spitze deutlich überragt; eine Eigenthümlichkeit, die ich bei keinem anderen mir bekannten Aegus wahrnahm. Uebrigens wäre es nicht unmöglich, dafs der Käfer die var. max. des von Hrn. Ritsema in den Notes from the Leyden Museum IV, 1882, p. 404 beschriebenen Aegus Leeuweni sein könnte, da die Unterschiede, die sich in der Stellung des zweiten Mandibelzahnes und der Sculptur zeigen, sich wohl auf die Gröfsenverschiedenheit (der Ritsema'sche Käfer mifst nur 27 mill.) zurückführen lassen; jedoch halte ich das nicht für wahrscheinlich, da in der sonst sehr umfafsenden und sorgfältigen Beschreibung des Leeuweni der Hinterwinkel des Vorderrückens nicht gedacht wird, also wohl angenommen werden mufs, dafs sie ohne Auszeichnung sind.

Ein einzelnes Stück jetzt in meiner Sammlung.

Bezeichnung immer nur auf die eine Seite pafst. Nennt man dann die an den beiden Enden der Ausbuchtung entstehenden Winkel: Ecken, versuras, so ist man in der Lage, die zu beschreibenden Formen mit wenigen Worten bestimmt und genau beschreiben zu können. Auch habe ich mich dieser Terminologie schon vorhin bedient.

Hannover, im December 1888.

G. Albers. 


\section{$2 \mathrm{BHL}$ Biodiversity Heritage Library}

Albers, G. 1889. "Beitrag zur Kenntnifs der Lucaniden-Fauna von Sumatra." Deutsche entomologische Zeitschrift 1889(2), 232-240.

https://doi.org/10.1002/mmnd.48018890203.

View This Item Online: https://www.biodiversitylibrary.org/item/103677

DOI: https://doi.org/10.1002/mmnd.48018890203

Permalink: https://www.biodiversitylibrary.org/partpdf/235442

\section{Holding Institution}

Harvard University, Museum of Comparative Zoology, Ernst Mayr Library

\section{Sponsored by}

Harvard University, Museum of Comparative Zoology, Ernst Mayr Library

\section{Copyright \& Reuse}

Copyright Status: Public domain. The BHL considers that this work is no longer under copyright protection.

This document was created from content at the Biodiversity Heritage Library, the world's largest open access digital library for biodiversity literature and archives. Visit BHL at https://www.biodiversitylibrary.org. 